\title{
Continued Fractions and Dynamics
}

\author{
Stefano Isola \\ Dipartimento di Matematica e Informatica, Università degli Studi di Camerino, Camerino Macerata, Italy \\ Email: stefano.isola@unicam.it
}

Received 17 January 2014; revised 17 February 2014; accepted 24 February 2014

Copyright (c) 2014 by author and Scientific Research Publishing Inc.

This work is licensed under the Creative Commons Attribution International License (CC BY).

http://creativecommons.org/licenses/by/4.0/

(c) (i) 0 pen Access

\section{Abstract \\ Several links between continued fractions and classical and less classical constructions in dynam- ical systems theory are presented and discussed.}

\section{Keywords}

Continued Fractions, Fast and Slow Convergents, Irrational Rotations, Farey and Gauss Maps, Transfer Operator, Thermodynamic Formalism

\section{Introduction}

The connection between Number Theory and Dynamical Systems Theory is receiving recently a considerable attention. In this paper, we review some aspects of this connection focusing on the interplay between continued fractions and one dimensional dynamics. In Section 2, we review some known facts about fast and slow convergents, highlighting their relations both with irrational rotation dynamics and the ergodic theory of the Gauss map. In Section 3, after recalling the construction and the basic properties of the Farey tree, we describe different ways of coding the paths on it, as well as their dynamical counterparts obtained by combining fractional linear transformations. Deeper insights into these connections are provided by the Minkowski question mark function, whose properties are discussed in Section 4. Finally, in Section 5, we present some applications of the thermodynamical formalism based on the previous constructions.

\section{Fast and Slow Convergents}

We start by reviewing some well known facts about continued fractions ${ }^{1}$.

Let

${ }^{1}$ Good general sources on this subject are [1]-[3]. 


$$
x=\frac{1}{a_{1}+\frac{1}{a_{2}+\frac{1}{a_{3}+\cdots}}} \equiv\left[a_{1}, a_{2}, a_{3}, \cdots\right]
$$

be the continued fraction expansion of the number $x \in[0,1]$. By applying Euclid's algorithm one sees that the above expansion terminates if and only if $x$ is a rational number. For $x$ irrational one can construct recursively a sequence $p_{n} / q_{n}$ of rational approximants of $x$ as

$$
p_{0} q_{0}=\frac{0}{1}, p_{1} q_{1}=\frac{1}{a_{1}} \text { and } \frac{p_{n+1}}{q_{n+1}}=\frac{a_{n+1} p_{n}+p_{n-1}}{a_{n+1} q_{n}+q_{n-1}}, \quad n \geq 1
$$

We can write this recursion in matrix form as follows: letting

$$
A:=\left(\begin{array}{ll}
1 & 0 \\
1 & 1
\end{array}\right) \text { and } B:=\left(\begin{array}{ll}
1 & 1 \\
1 & 0
\end{array}\right)
$$

and noting that

$$
B A^{k-1}=\left(\begin{array}{ll}
k & 1 \\
1 & 0
\end{array}\right)
$$

we have

$$
\left(\begin{array}{ll}
p_{1} & p_{0} \\
q_{1} & q_{0}
\end{array}\right)=A^{a_{1}}
$$

and

$$
\left(\begin{array}{cc}
p_{n+1} & p_{n} \\
q_{n+1} & q_{n}
\end{array}\right)=A^{a_{1}} B A^{a_{2}-1} \cdots B A^{a_{n+1}-1}, \quad n \geq 1
$$

A short manipulation of (2.2) gives $q_{n+1} p_{n}-q_{n} p_{n+1}=-\left(q_{n} p_{n-1}-q_{n-1} p_{n}\right)$. Since $q_{1} p_{0}-q_{0} p_{1}=-1$ one obtains inductively the Lagrange formula

$$
q_{n} p_{n-1}-q_{n-1} p_{n}=(-1)^{n}, \quad n \geq 1
$$

Another useful formula which can be easily obtained from (2.2) is the following: for all $r \geq 1$ and $n \geq 1$,

$$
\left[a_{1}, a_{2}, a_{3}, \cdots, a_{n}+\frac{1}{r}\right]=\frac{r p_{n}+p_{n-1}}{r q_{n}+q_{n-1}} .
$$

Letting $r \rightarrow \infty$ we get in particular

$$
\left[a_{1}, a_{2}, \cdots, a_{n}\right]=\frac{p_{n}}{q_{n}} .
$$

Note that

$$
\frac{q_{n-1}}{q_{n}}=\frac{1}{a_{n}+\frac{1}{\frac{q_{n-2}}{q_{n-1}}}}=\frac{1}{a_{n}+\frac{1}{a_{n-1}+\frac{1}{\frac{q_{n-3}}{q_{n-2}}}}}
$$

and so forth. We thus have the so called mirror formula (some consequences of which have been investigated in [4]):

$$
\text { If } \frac{p_{n}}{q_{n}}=\left[a_{1}, a_{2}, \cdots, a_{n}\right] \text { then } \frac{q_{n-1}}{q_{n}}=\left[a_{n}, a_{n-1}, \cdots, a_{1}\right]
$$


The numbers $\frac{p_{n}}{q_{n}}$ are called continued fraction convergents (CFC) of $x$ and it turns out that the $n$-th CFC $\frac{p_{n}}{q_{n}}$ is the best rational approximation to $x$ whose denominator does not exceed $q_{n}$ [2]. One sees that

$$
\frac{p_{2 n}}{q_{2 n}}<x<\frac{p_{2 n-1}}{q_{2 n-1}}, \quad \forall n>0 .
$$

Putting $r=a_{n+1}$ in (2.8) we get

$$
\left[a_{1}, a_{2}, a_{3}, \cdots, a_{n}+\frac{1}{a_{n+1}}\right] \equiv\left[a_{1}, a_{2}, a_{3}, \cdots, a_{n}, a_{n+1}\right]=\frac{p_{n+1}}{q_{n+1}} .
$$

But what happens if $r$ in (2.8) takes on an intermediate value $1,2, \cdots, a_{n+1}$ ?

Definition 2.1 For $n \geq 1$ the sets $\left\{\frac{r p_{n}+p_{n-1}}{r q_{n}+q_{n-1}}\right\}$ for $1 \leq r \leq a_{n+1}$ are the n'th Farey convergents (FC) for the real number $x \in[0,1)$.

Example. Let $x=e-2=[1,2,1,1,4,1,1,6, \cdots]$. The first five CFC are

$$
\begin{array}{ll}
n=1 \quad \frac{p_{1}}{q_{1}}=1 \\
n=2 \quad \frac{p_{2}}{q_{2}}=\frac{1}{1+\frac{1}{2}}=\frac{2}{3} \\
n=3 \quad \frac{p_{3}}{q_{3}}=\frac{1}{1+\frac{1}{2+\frac{1}{1}}=\frac{3}{4}} \\
n=4 \quad \frac{p_{4}}{q_{4}}=\frac{1}{1+\frac{1}{2+\frac{1}{1+\frac{1}{1}}}}=\frac{5}{7} \\
n=5 \quad \frac{5}{q_{5}}=\frac{1}{1+\frac{1}{2+\frac{1}{1+\frac{1}{1+\frac{1}{4}}}}}=\frac{23}{32}
\end{array}
$$

On the other hand, within the same accuracy, there are $2+1+1+4=8$ FC's. They are

$$
\begin{array}{ll}
n=1, r=1, & \frac{t_{1,1}}{s_{1,1}}=\frac{p_{1}+p_{0}}{q_{1}+q_{0}}=\frac{1}{2} \\
n=1, r=2, & \frac{t_{1,2}}{s_{1,2}}=\frac{2 p_{1}+p_{0}}{2 q_{1}+q_{0}}=\frac{2}{3} \\
n=2, r=1, & \frac{t_{2,1}}{s_{2,1}}=\frac{p_{2}+p_{1}}{q_{2}+q_{1}}=\frac{3}{4}
\end{array}
$$




$$
\begin{aligned}
& n=3, r=1, \quad \frac{t_{3,1}}{s_{3,1}}=\frac{p_{3}+p_{2}}{q_{3}+q_{2}}=\frac{5}{7} \\
& n=4, r=1, \quad \frac{t_{4,1}}{s_{4,1}}=\frac{p_{4}+p_{3}}{q_{4}+q_{3}}=\frac{8}{11} \\
& n=4, r=2, \quad \frac{t_{4,2}}{s_{4,2}}=\frac{2 p_{4}+p_{3}}{2 q_{4}+q_{3}}=\frac{13}{18} \\
& n=4, r=3, \quad \frac{t_{4,3}}{s_{4,3}}=\frac{3 p_{4}+p_{3}}{3 q_{4}+q_{3}}=\frac{18}{25} \\
& n=4, r=4, \quad \frac{t_{4,4}}{s_{4,4}}=\frac{4 p_{4}+p_{3}}{4 q_{4}+q_{3}}=\frac{23}{32}
\end{aligned}
$$

We now need some notions.

Definition 2.2 The Farey sum over two rationals $\frac{a}{b}$ and $\frac{a^{\prime}}{b^{\prime}}$ is the mediant operation given by

$$
\frac{a}{b} \oplus \frac{a^{\prime}}{b^{\prime}}:=\frac{a+a^{\prime}}{b+b^{\prime}}=\frac{a^{\prime \prime}}{b^{\prime \prime}}
$$

It is easy to see that $\frac{a^{\prime \prime}}{b^{\prime \prime}}$ falls in the interval $\left(\frac{a}{b}, \frac{a^{\prime}}{b^{\prime}}\right)^{2}$. We say that $\frac{a}{b}$ and $\frac{a^{\prime}}{b^{\prime}}$ are Farey neighbours if $a b^{\prime}-a^{\prime} b= \pm 1$. Two Farey neighbours define a Farey interval and each Farey interval can be labeled uniquely according to the mediant (child) $\frac{a^{\prime \prime}}{b^{\prime \prime}}=\frac{a+a^{\prime}}{b+b^{\prime}}$ of the neighbours.

Observe that given a pair of consecutive FC's, say

$$
\frac{t_{n, r}}{s_{n, r}}=\frac{r p_{n}+p_{n-1}}{r q_{n}+q_{n-1}} \text { and } \frac{t_{n, r+1}}{s_{n, r+1}}=\frac{(r+1) p_{n}+p_{n-1}}{(r+1) q_{n}+q_{n-1}}
$$

for some $n \geq 1$ and $1 \leq r<a_{n+1}$, we have

$$
\frac{t_{n, r+1}}{S_{n, r+1}}=\frac{t_{n, r}}{S_{n, r}} \oplus \frac{p_{n}}{q_{n}}
$$

Moreover

$$
\frac{q_{n}}{t_{n, r}}-\frac{p_{n}}{S_{n, r}}=\frac{q_{n}}{p_{n-1}}-\frac{p_{n}}{q_{n-1}}=(-1)^{n}
$$

by Lagrange's formula. Therefore, for every $n \geq 1$, each FC $\frac{t_{n, r}}{s_{n, r}}$ for $r=1, \cdots, a_{n+1}$ is a Farey neighbour of $\frac{p_{n}}{q_{n}}$, the corresponding Farey interval getting smaller and smaller as $r$ increases. More precisely, using again Lagrange's formula, one easily obtains

$$
\left|\frac{p_{n}}{q_{n}}-\frac{r p_{n}+p_{n-1}}{r q_{n}+q_{n-1}}\right|=\frac{1}{q_{n}\left(r q_{n}+q_{n-1}\right)}
$$

We therefore see that the FC $\frac{t_{n, r}}{s_{n, r}}$ is the best one-sided rational approximation to $x$ whose denominator

\footnotetext{
${ }^{2}$ The origin of these names traces back to Cauchy, who proved this property after it was observed by John Farey in 1816 [5], and named "Farey series" the numbers obtained in this way.
} 
does not exceed $s_{n, r}$ (although, if $r<a_{n+1}$, there might be a CFC with denominator less than $s_{n, r}$ and closer to $X$ on the other side of $x$ ). Increasing $r$, once we arrive at $r=a_{n+1}$ we hit a new CFC on the current side of $x$, closer than the previous CFC. Finally, using matrix notation, the FC's can be expressed in terms of intermediate products in (2.5) for $n \geq 1$ as

$$
\left(\begin{array}{ll}
t_{n, r} & p_{n} \\
s_{n, r} & q_{n}
\end{array}\right)=A^{a_{1}} B A^{a_{2}-1} \cdots B A^{a_{n}-1} B A^{r-1}, \quad 1 \leq r \leq a_{n+1} .
$$

The algorithm which produces the sequence of $F C$ 's of a given real number is called slow continued fraction algorithm (see, e.g., [6] [7]).

Remark 2.3 The set $\mathcal{F}_{\ell}$ of Farey fractions of order $\ell$ is the set of irreducible fractions in $[0,1]$ with denominator $\leq \ell$, listed in order of magnitude (see [8]). Thus, $\tilde{\mathcal{F}}_{1}=\left(\frac{0}{1}, \frac{1}{1}\right)$,

$$
\tilde{\mathcal{F}}_{2}=\left(\frac{0}{1}, \frac{1}{2}, \frac{1}{1}\right), \quad \tilde{\mathcal{F}}_{3}=\left(\frac{0}{1}, \frac{1}{3}, \frac{1}{2}, \frac{2}{3}, \frac{1}{1}\right), \quad \tilde{\mathcal{F}}_{4}=\left(\frac{0}{1}, \frac{1}{4}, \frac{1}{3}, \frac{1}{2}, \frac{2}{3}, \frac{3}{4}, \frac{1}{1}\right)
$$

and so on. In particular $\left|\tilde{\mathcal{F}}_{\ell}\right|-2=\sum_{k=1}^{\ell} \varphi(k) \sim \frac{3 \ell^{2}}{\pi^{2}}$ with Euler totient function

$\varphi(k)=|\{0<i \leq k: \operatorname{gcd}(i, k)=1\}|$. Then we see that each $\frac{t_{n, r}}{s_{n, r}}$ for $r=1, \cdots, a_{n+1}$ is consecutive to $\frac{p_{n}}{q_{n}}$ in $\tilde{\mathcal{F}}_{\ell}$ for $s_{n, r}<\ell \leq s_{n, r+1}$.

\subsection{Connection to Rotations of the Circle}

One can interpret the above construction in terms of a kind of renormalization procedure for rotations of the circle $[0,1)$ through an angle $x$. With no loss we take the initial point to be the origin 0 and set $J_{0}=[0, x]$. Since $a_{1}=\left[\frac{1}{x}\right]$ we have $a_{1} x \leq 1<\left(a_{1}+1\right) x$ and thus

$$
1=a_{1}\left|J_{0}\right|+\left|J_{1}\right|
$$

with

$$
\left|J_{1}\right|=1-a_{1} x=p_{1}-x q_{1}
$$

Moreover we have

$$
\frac{\left|J_{1}\right|}{\left|J_{0}\right|}=\frac{1}{x}-a_{1}=\left[a_{2}, a_{3}, \cdots\right]
$$

and therefore $a_{2}\left|J_{1}\right| \leq\left|J_{0}\right|<\left(a_{2}+1\right)\left|J_{1}\right|$ or, which is the same,

$$
\left|J_{0}\right|=a_{2}\left|J_{1}\right|+\left|J_{2}\right|
$$

with

$$
\left|J_{2}\right|=x-a_{2}\left(1-a_{1} x\right)=q_{2} x-p_{2} .
$$

Iterating this procedure, we construct a family of nested intervals (see Figure 1) $J_{n}, n \geq 0$, such that

$$
\frac{\left|J_{n}\right|}{\left|J_{n-1}\right|}=\left[a_{n+1}, a_{n+2}, \cdots\right]
$$

and

$$
\left|J_{n-1}\right|=a_{n+1}\left|J_{n}\right|+\left|J_{n+1}\right|, \quad n \geq 0,
$$

where we have set $J_{-1}=[0,1]$. Using (2.18), (2.19) and (2.21) one gets inductively the formula 


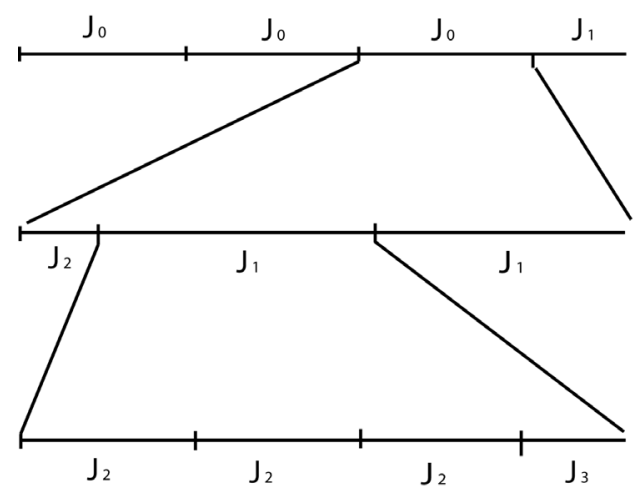

Figure 1. The construction of nested intervals.

$$
f_{n}:=\left|J_{n}\right|=\left|q_{n} x-p_{n}\right|=(-1)^{n}\left(q_{n} x-p_{n}\right) .
$$

Note that

$$
1=q_{n} f_{n-1}+q_{n-1} f_{n}, \quad \forall n \geq 1 .
$$

Now, if we denote by $d$ the euclidean metric on $[0,1)$ then

$$
d\left(0, T^{r} 0\right)=\min _{p \in \mathbb{Z}}|r x-p|=:\|r x\| .
$$

Therefore

$$
d\left(0, T^{q_{n}} 0\right)=\left\|q_{n} x\right\|=\left|q_{n} x-p_{n}\right|=f_{n}
$$

That is, the sequence of arc-lengths $f_{n}$ is but the sequence of successive closest distances to the initial point. This can be seen in the following way: starting from 0 and iterating $a_{1}$ times one ends up at the point $a_{1} x=q_{1} x \quad$ which lies on the left of 0 and is the point closest to 0 up to now, being distant $\left|J_{1}\right|$ from it. Iterating $a_{1}$ more times one ends up at the point $2 a_{1} x=2 q_{1} x$ which lies on the left of 0 at distance $2\left|J_{1}\right|, \ldots$ iterating $a_{2} a_{1}$ times one ends up at the point $a_{2} a_{1} x=a_{2} q_{1} x=\left(q_{2}-1\right) x$ which still lies on the left of 0 , at distance $a_{2}\left|J_{1}\right|$. One more iterate yields the point $q_{2} x$ which now lies on the right of 0 at distance $\left|J_{2}\right|$ and is the point closest to 0 up to now, and so on and so forth (for more details see [9]). The above implies that the first return map in the interval $J_{n}$ (which is $\left[0, f_{n}\right]$ or $\left[1-f_{n}, 1\right]$ according whether $n$ is even or odd) is the rotation through the angle $(-1)^{n+1} f_{n+1}=q_{n+1} x-p_{n+1}$. Finally, one has the equivalence:

$$
f_{n} \approx 0 \Leftrightarrow x \approx\left[a_{1}, \cdots, a_{n}\right]=\frac{p_{n}}{q_{n}} .
$$

In addition, for each $r=1, \cdots, a_{n+1}$, it holds

$$
f_{n} \approx f_{n-1} r \Leftrightarrow x \approx\left[a_{1}, \cdots, a_{n}+\frac{1}{r}\right]=\frac{t_{n, r}}{s_{n, r}} .
$$

The three distance theorem. The points $\{k \alpha\}$ with $0 \leq k \leq \ell$ partition the unit circle into $\ell+1$ intervals. A classical result (see e.g. [10]), which can be easily obtained by induction using the above construction, is that the possible lengths of these intervals are organized according to the Farey convergents in the following way:

- If $0<\ell \leq q_{1}$ then there are two distinct lengths: $f_{0}$ and $1-\ell f_{0}$ (which become $f_{0}$ and $f_{1}$ when $\left.\ell=q_{1}\right)$.

- If $r q_{n}+q_{n-1} \leq \ell<(r+1) q_{n}+q_{n-1}$ for some $n \geq 1$ and $1 \leq r \leq a_{n+1}$ then there are at most three lengths:

$f_{n}, f_{n-1}-r f_{n}$ and $f_{n-1}-(r-1) f_{n}$, the last of which disappears when $\ell=(r+1) q_{n}+q_{n-1}-1$.

We point out that in the second case above there are two intervals, chosen from among those having the smallest lengths:

$$
f_{n}=\left|q_{n} x-p_{n}\right| \text { and } f_{n-1}-r f_{n}=\left|\left(r q_{n}+q_{n-1}\right) x-\left(r p_{n}+p_{n-1}\right)\right|
$$


which have 0 as their common endpoint. We then see that the approximations (26) and (27) are the same as shrinking one of these intervals to zero. Moreover, the fractions $\frac{p_{n}}{q_{n}}$ and $\frac{r p_{n}+p_{n-1}}{r q_{n}+q_{n-1}}$ are the two successive elements of $\tilde{\mathcal{F}}_{n}$ having $x$ between them (see also Remark 2.3).

\subsection{Growth of Denominators}

The Gauss map $G:[0,1] \rightarrow[0,1]$ is defined as

$$
G(x)=\{1 / x\} \quad \text { for } x>0 \text { and } G(0)=0 .
$$

It is well known that $G$ has an a.c. invariant ergodic probability measure $\mu$ given by

$$
\mu(\mathrm{d} x)=\frac{1}{\log 2} \frac{\mathrm{d} x}{(1+x)}
$$

A short reflection shows that $x=\left[a_{1}, a_{2}, \cdots, a_{n}+G^{n}(x)\right]$ or else

$$
x=\frac{\left(G^{n}(x)\right)^{-1} p_{n}+p_{n-1}}{\left(G^{n}(x)\right)^{-1} q_{n}+q_{n-1}} .
$$

From this we obtain at once

$$
G^{n}(x)=-\frac{q_{n} x-p_{n}}{q_{n-1} x-p_{n-1}}=-\frac{f_{n}}{f_{n-1}},
$$

where the numbers $f_{n}$ have been introduced in (2.22). Therefore

$$
f_{n}=\prod_{k=0}^{n} G^{k}(x)
$$

and, by the ergodic theorem, we have for $\mu$-almost all $x \in[0,1]$ and then almost everywhere,

$$
\lim _{n \rightarrow \infty} \frac{1}{n} \log f_{n}=\int_{0}^{1} \log x \mu(\mathrm{d} x)=-\frac{\pi^{2}}{12 \log 2} .
$$

Since $\left[\left(G^{n}(x)\right)^{-1}\right]=a_{n+1}$ and thus $a_{n+1}<\left(G^{n}(x)\right)^{-1}<a_{n+1}+1$ another consequence of (2.30) is that

$$
\frac{1}{a_{n+1}+2}<\frac{q_{n}}{q_{n}+q_{n+1}}<q_{n} f_{n}<\frac{q_{n}}{q_{n+1}}<\frac{1}{a_{n+1}}
$$

and therefore using (2.31)

$$
\frac{1}{2}<q_{n} f_{n-1}<1 .
$$

Putting together (2.32) and (2.33) we get the classical theorem of Lévy

$$
\frac{\log q_{n}}{n} \rightarrow \frac{\pi^{2}}{12 \log 2} \text { almost everywhere }
$$

On the other hand we may expect the growth of FC's denominator to be subexponential. Indeed, let $\frac{t_{m}}{s_{m}} \equiv \frac{t_{n, r}}{s_{n, r}}$ with $m=\sum_{k=2}^{n} a_{k}+r$ be the $m$-th FC. Its denominator satisfies $q_{n}<s_{m} \leq q_{n+1}$. It is a result of Khinchin and Lévy (see [1]) that

$$
\frac{1}{n \log n} \sum_{k=1}^{n} a_{k} \rightarrow \frac{1}{\log 2} \text { in measure }
$$


Combining the above we get the following

Lemma 2.4

$$
\frac{\log s_{m}}{m} \sim \frac{\pi^{2}}{12 \log m} \text { in measure }
$$

Of course there are special behaviours: take $x=(\sqrt{5}-1) / 2=[1,1,1, \cdots]$, then $s_{n}=q_{n}$ and both are equal to the $n$-th Fibonacci number. Hence $n^{-1} \log q_{n}$ converge to $x^{-1}$.

\section{A Walk on the Farey Tree}

Having fixed $\ell \geq 1$, let $\mathcal{F}_{\ell}$ be the ascending sequence of irreducible fractions between 0 and 1 constructed inductively in the following way: set first $\mathcal{F}_{1}=\left(\frac{0}{1}, \frac{1}{1}\right)$, then $\mathcal{F}_{\ell}$ is obtained from $\mathcal{F}_{\ell-1}$ by inserting among each pair of neighbours $\frac{a}{b}$ and $\frac{a^{\prime}}{b^{\prime}}$ in $\mathcal{F}_{\ell-1}$ their child $\frac{a^{\prime \prime}}{b^{\prime \prime}}$ as in (2.13). Thus

$$
\mathcal{F}_{2}=\left(\frac{0}{1}, \frac{1}{2}, \frac{1}{1}\right), \quad \mathcal{F}_{3}=\left(\frac{0}{1}, \frac{1}{3}, \frac{1}{2}, \frac{2}{3}, \frac{1}{1}\right), \quad \mathcal{F}_{4}=\left(\frac{0}{1}, \frac{1}{4}, \frac{1}{3}, \frac{2}{5}, \frac{1}{2}, \frac{3}{5}, \frac{2}{3}, \frac{3}{4}, \frac{1}{1}\right)
$$

and so on. The elements of $\mathcal{F}_{\ell}$ are called again Farey fractions. Evidently $\mathcal{F}_{\ell} \supseteq \tilde{\mathcal{F}}_{\ell}$.

Remark 3.1 It has been shown in ([11], Thm 2.6) that the set $\left\{\{\log b\} \mid \frac{a}{b} \in F_{\ell}\right\}$ becomes equidistributed as $\ell \rightarrow \infty$. More specifically, the probability measure $\frac{1}{2^{\ell}+1} \sum_{a b \in \mathcal{F}_{\ell+1}} \delta_{\{\log b\}}$ converges to the Lebesgue measure on $[0,1]$.

Definition 3.2 For $\ell \geq 1$ we say that a Farey fraction $x$ has rank $\ell$ if $x \in \mathcal{F}_{\ell+1} \backslash \mathcal{F}_{\ell}$.

We also define the $\operatorname{rank}\left(\frac{0}{1}\right)=\operatorname{rank}\left(\frac{1}{1}\right)=0$. For $\ell \geq 1$ there are exactly $2^{\ell-1}$ Farey fractions of rank $\ell$ and their sum is equal to $2^{\ell-2}$. Recall that every rational number $x \in(0,1)$ has a unique finite continued fraction expansion $x=\left[a_{1}, \cdots, a_{n}\right]$ with $a_{n}>1$ [2]. The validity of the following relation will arise straightforwardly in the sequel:

\section{Lemma 3.3}

$$
x=\left[a_{1}, \cdots, a_{n}\right] \rightarrow \operatorname{rank}(x)=\sum_{i=1}^{n} a_{i}-1
$$

Remark 3.4 Note that, according to the above Lemma, the cardinality $2^{\ell-1}$ of $\mathcal{F}_{\ell+1} \backslash \mathcal{F}_{\ell}$ can be interpreted as the number of choices of integers $a_{1}, \cdots, a_{n}$, with $1 \leq n \leq \ell$ and so that $a_{i} \geq 1$ for $i=1, \cdots, n-1, a_{n}>1$ and $\sum_{i=1}^{n} a_{i}=\ell+1$. Indeed, for each fixed $n$ the number of such choices is $\left(\begin{array}{l}\ell-1 \\ n-1\end{array}\right)$, then sum over $n=1, \cdots, \ell$.

It is also easy to realize that all Farey fractions which fall in the interval $\left(\frac{1}{\ell+1}, \frac{1}{\ell}\right)$ have rank greater than or equal to $\ell+1$, whereas their continued fraction expansion starts with $a_{1}=\ell$.

An interesting object is the Farey tree $\mathcal{F}$ whose vertex-set is $\mathbb{Q} \cap(0,1)$ and which is constructed as follows (see Figure 2):

- every column in $\mathcal{F}$ contains one entry (vertex or node);

- for $\ell \geq 1$ the $\ell$-th row is $\mathcal{F}_{\ell+1} \backslash \mathcal{F}_{\ell}$;

- the node $\frac{a+a^{\prime}}{b+b^{\prime}}$, representing the interval $\left[\frac{a}{b}, \frac{a^{\prime}}{b^{\prime}}\right]$, is connected by edges to its left child $\frac{2 a+a^{\prime}}{2 b+b^{\prime}}$ and right child $\frac{a+2 a^{\prime}}{b+2 b^{\prime}}$ in the underlying row. 


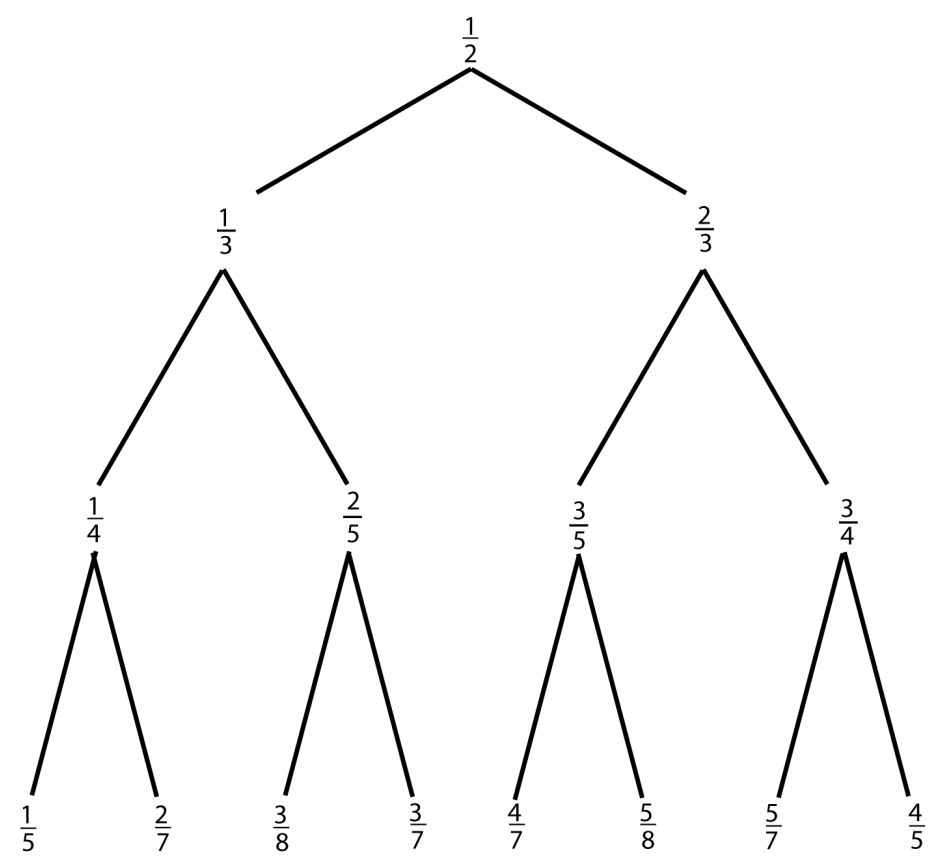

Figure 2. The first four levels of the Farey tree.

Note that the fractions $\frac{0}{1}$ and $\frac{1}{1}$ play the role of ancestors when using the Farey sum to obtain one row from the previous one. Besides the Farey sum, an alternative way to construct recursively the entries of $\mathcal{F}$ is as follows.

Definition 3.5 Given $\frac{a}{b} \in \mathcal{F}$ its descendants are the symmetrical entries of $\mathcal{F}$ given by $\frac{a}{a+b}$ and $\frac{b}{a+b}$, respectively.

Lemma 3.6 The collection of all descendants of the entries of a given row in $\mathcal{F}$ is precisely the underlying row.

Proof. If $\frac{a}{b}=\left[a_{1}, \cdots, a_{n}\right]$ then $\frac{a}{a+b}=\left[a_{1}+1, \cdots, a_{n}\right]$ and $\frac{b}{a+b}=\left[1, a_{1}, \cdots, a_{n}\right]$. Therefore $\operatorname{rank}\left(\frac{a}{a+b}\right)=\operatorname{rank}\left(\frac{b}{a+b}\right)=\operatorname{rank}\left(\frac{a}{b}\right)+1$ and the claim follows.

Remark 3.7 If $\frac{a}{b}=\left[a_{1}, a_{2} \cdots, a_{n}\right]$ and $\frac{a^{\prime}}{b^{\prime}}=\left[a_{n}, a_{n-1}, \cdots, a_{1}\right]$ then $\operatorname{rank}\left(\frac{a}{b}\right)=\operatorname{rank}\left(\frac{a^{\prime}}{b^{\prime}}\right)$ and $b=b^{\prime}$.

\subsection{The $\{L, R\}$ Coding}

Every rational number in $(0,1)$ appears exactly once in the above construction and corresponds to a unique finite path on $\mathcal{F}$ starting at the root node $\frac{1}{2}$ and whose number of vertices equals the rank of the rational number. We can code this path in the following way: first, any $\frac{l}{r} \in \mathcal{F}$ can be uniquely decomposed as ${ }^{3}$

$$
\frac{l}{r}=\frac{m}{s} \oplus \frac{n}{t} \quad \text { with } n s-m t=1
$$

and the unimodular relations

${ }^{3}$ All fractions are supposed in lowest terms. 


$$
s l-r m=r n-l t=n s-m t=1
$$

plainly hold. The neighbours $\frac{m}{s}$ and $\frac{n}{t}$ are thus the 'parents' of $\frac{l}{r}$ in $\mathcal{F}$ and we may accordingly identify

$$
\frac{l}{r} \Leftrightarrow\left(\begin{array}{cc}
n & m \\
t & s
\end{array}\right) \in \mathcal{U}
$$

with

$$
\mathcal{U}=\left\{\left(\begin{array}{ll}
a & b \\
c & d
\end{array}\right) \mid 0<a \leq c, 0 \leq b<d, a d-b c=1\right\} .
$$

Note that the left column bears on the right parent and viceversa. Thus

$$
\frac{1}{2} \Leftrightarrow\left(\begin{array}{ll}
1 & 0 \\
1 & 1
\end{array}\right)
$$

On the other hand, any $\frac{l}{r}$ as above has a unique pair of (left and right) children, given by

$$
\frac{m}{s} \oplus \frac{m+n}{s+t} \text { and } \frac{m+n}{s+t} \oplus \frac{n}{t}
$$

respectively. In order to generate them we set

$$
L:=\left(\begin{array}{ll}
1 & 0 \\
1 & 1
\end{array}\right) \text { and } R:=\left(\begin{array}{ll}
1 & 1 \\
0 & 1
\end{array}\right)
$$

Note that for $k \in \mathbb{Z}$

$$
L^{k}:=\left(\begin{array}{ll}
1 & 0 \\
k & 1
\end{array}\right) \text { and } R^{k}:=\left(\begin{array}{ll}
1 & k \\
0 & 1
\end{array}\right)
$$

and also

$$
R^{k} S=S L^{k} \quad \text { with } S=S^{-1}=\left(\begin{array}{ll}
0 & 1 \\
1 & 0
\end{array}\right)
$$

Moreover, we have

$$
\left(\begin{array}{cc}
n & m \\
t & s
\end{array}\right)\left(\begin{array}{ll}
1 & 0 \\
1 & 1
\end{array}\right)=\left(\begin{array}{cc}
m+n & m \\
s+t & s
\end{array}\right) \Leftrightarrow \frac{m}{s} \oplus \frac{m+n}{s+t}
$$

and

$$
\left(\begin{array}{cc}
n & m \\
t & s
\end{array}\right)\left(\begin{array}{ll}
1 & 1 \\
0 & 1
\end{array}\right)=\left(\begin{array}{cc}
n & m+n \\
t & s+t
\end{array}\right) \Leftrightarrow \frac{m+n}{s+t} \oplus \frac{n}{t}
$$

In other words, the matrices $L$ and $R$, when acting from the right, move to the left and right child in $\mathcal{F}$, respectively. Moreover, it is plain that given $Y \in \mathcal{V}$ we have $Y R \in \mathcal{V}$ and $Y L \in \mathcal{V}$. We have thus proved the following

Proposition 3.8 To each entry $x \in \mathcal{F}$ there corresponds a unique element $X \in \mathcal{U}$ which, in turn, can be uniquely presented as

$$
X=L \prod_{i} M_{i}
$$

where the number of terms in the product $L \prod_{i} M_{i}$ is equal to $\operatorname{rank}(x)$ and $M_{i}=L$ or $M_{i}=R$ according whether the $i$-th turn, along the descending path in $\mathcal{F}$ which starts from the root node $\frac{1}{2}$ and reaches $x$, goes left or right. 
Remark 3.9 By the way, the matrices $L$ and $R$ induce the so called Farey tesselation of the upper half plane $H=\{z: \operatorname{Im} z>0\} \quad$ (see [12]).

Example. $\frac{3}{10}$ is the right child of $\frac{2}{7}$, which is the right child of $\frac{1}{4}$, which is the left child of $\frac{1}{3}$, which is the left child of $\frac{1}{2}$. Thus

$$
\frac{3}{10} \Leftrightarrow\left(\begin{array}{ll}
1 & 2 \\
3 & 7
\end{array}\right)=L L L R R
$$

For $\frac{3}{11}$, which is the left child of $\frac{2}{7}$, we find

$$
\frac{3}{11} \Leftrightarrow\left(\begin{array}{ll}
2 & 1 \\
7 & 4
\end{array}\right)=L L L R L
$$

Note that $\operatorname{rank}\left(\frac{3}{10}\right)=\operatorname{rank}\left(\frac{3}{11}\right)=5$.

To any given irrational number $x \in[0,1]$ we may associate a unique infinite path on $\mathcal{F}$, and thus a unique semi-infinite word in $\{L, R\}^{\mathbb{N}}$. Bearing in mind the continued fraction expansion (2.1) of $x$, let

$$
\frac{t_{1,1}}{s_{1,1}}=\frac{1}{a_{1}+1}
$$

the first FC of $x$. In order to reach it from the top of $\mathcal{F}$ we need the block $L^{a_{1}-1}$. Whence we code $x$ through the map $\phi:[0,1] \rightarrow\{L, R\}^{\mathbb{N}}$ defined by

$$
\phi(x)=L^{a_{1}} M_{1} M_{2} \cdots
$$

where $M_{i}=L$ or $M_{i}=R$ according whether the $i$-th turn along the infinite path in $\mathcal{F}$ which starts from $\frac{t_{1,1}}{s_{1,1}}$ and approaches $x$ along the sequence of successive FC's goes left or right. This coding is faithful to the binary structure of $\mathcal{F}$ but apparently not so much to the continued fraction expansion of $x$. To make the latter more transparent we may note that, according to the characterization of the FC's given above (see (2.15) and (2.16)), the symbols $L$ and $R$ in (3.13) come in blocks whose lengths are given by nothing but the partial quotients $a_{i}$ of $x$. More precisely, a short reflection shows that the following rule is in force: the first block is such that $M_{i}=R$ if $1 \leq i \leq a_{2}$. Moreover, for $k \geq 2$ let

then we have

$$
b_{k}=\sum_{i=2}^{k} a_{i}
$$

$$
M_{i}= \begin{cases}L, & \text { if } b_{2 k-2}<i \leq b_{2 k-1}, \\ R, & \text { if } b_{2 k-1}<i \leq b_{2 k} .\end{cases}
$$

In other words, we have the coding

$$
x=\left[a_{1}, a_{2}, a_{3}, \cdots\right] \leftrightarrow \phi(x)=L^{a_{1}} R^{a_{2}} L^{a_{3}} \cdots
$$

Furthermore we set $\phi(0)=L^{\infty}$ and $\phi(1)=L R^{\infty}$. More generally, we note that each rational $x$ has two infinite paths which agree down to node $x$ : they are those starting with the finite sequence coding the path to reach $x$ from the root node and terminating with either $R L^{\infty}$ or $L R^{\infty}$. We shall agree that $\phi(x)$ terminates with $R L^{\infty}$ or $L R^{\infty}$ according whether the number of its (finite) partial quotients is even or odd. On the other hand, for notational simplicity' sake we shall assume this agreement only implicitly. We summarize the above in the following

Theorem 3.10 To $x \in[0,1]$ with continued fraction expansion $x=\left[a_{1}, a_{2}, a_{3}, \cdots\right]$ there corresponds a uni- 
que sequence $\phi(x) \in\{L, R\}^{\mathbb{N}}$ given by $\phi(x)=L^{a_{1}} R^{a_{2}} L^{a_{3}} \ldots$ which represents an infinite path on $\mathcal{F}$ whose sequence of vertices starting from the $a_{1}$-th is precisely the sequence $\left(t_{m} s_{m}\right)_{m>1}$ of FC's of $x$. Moreover, if $\succ$ denotes the lexicographic order on $\{L, R\}^{\mathbb{N}}$ then

$$
x>y \Rightarrow \phi(x) \succ \phi(y) .
$$

An simple consequence of the above construction is the following result.

Proposition 3.11 Let $x=\left[a_{1}, \cdots, a_{n}\right]$ with $a_{n}>1$ and $n$ even. Then its left and right children in $\mathcal{F}$ are given by $x^{\prime}=\left[a_{1}, \cdots, a_{n}-1,2\right]$ and $x^{\prime \prime}=\left[a_{1}, \cdots, a_{n}+1\right]$, respectively. If instead $n$ is odd the expansions for $x^{\prime}$ and $x^{\prime \prime}$ have to be interchanged.

Proof. Since $n$ is even we can write

$$
x=\left[a_{1}, \cdots, a_{n}\right] \leftrightarrow \phi(x)=L^{a_{1}} R^{a_{2}} \cdots R^{a_{n}-1} .
$$

Therefore

$$
x^{\prime}=\phi^{-1}\left(L^{a_{1}} R^{a_{2}} \cdots R^{a_{n}-1} L\right) \text { and } x^{\prime \prime}=\phi^{-1}\left(L^{a_{1}} R^{a_{2}} \cdots R^{a_{n}}\right)
$$

which yield the claim. A similar reasoning applies for $n$ odd.

\subsection{The $\{A, B\}$ Coding}

Using (3.9) we can write

$$
L^{a_{1}} R^{a_{2}} L^{a_{3}} \cdots=L^{a_{1}} S L^{a_{2}} S L^{a_{3}} \cdots
$$

On the other hand we have $L \equiv A$ and (see (2.4))

$$
S L^{k}=\left(\begin{array}{ll}
k & 1 \\
1 & 0
\end{array}\right)=B A^{k-1}
$$

This defines a recoding $\psi:[0,1] \rightarrow\{A, B\}^{\mathbb{N}}$ so that

$$
x=\left[a_{1}, a_{2}, a_{3}, \cdots\right] \leftrightarrow \psi(x)=A^{a_{1}} B A^{a_{2}-1} B A^{a_{3}-1} \cdots
$$

The FC $\frac{t_{n, r+1}}{s_{n, r+1}}$ of $x$, which has rank $\ell=\sum_{i=1}^{n} a_{i}+r$, will then be expressed as

$$
\phi\left(\frac{t_{n, r+1}}{s_{n, r+1}}\right)=\left\{\begin{array}{l}
L^{a_{1}} R^{a_{2}} \cdots L^{a_{n}} R^{r}, n \text { odd, } \\
L^{a_{1}} R^{a_{2}} \cdots R^{a_{n}} L^{r}, n \text { even, }
\end{array}\right.
$$

or else

$$
\psi\left(\frac{t_{n, r+1}}{s_{n, r+1}}\right)=A^{a_{1}} B A^{a_{2}-1} \cdots B A^{a_{n}-1} B A^{r-1} .
$$

Note that both expansions have exactly $\ell$ terms and the latter agrees with (2.17) once we interpret the l.h.s. of (2.17) as the FC $\frac{t_{n, r+1}}{s_{n, r+1}}$ of $x$, that is taking the Farey sum of the columns in the same spirit as (3.3).

Example. The example with $x=e-2=[1,2,1,1,4,1,1,6, \cdots]$ discussed above, which yields

$$
\phi(e-2)=L R R L R L L L L R L \cdots \quad \text { or else } \psi(e-2)=A B A B B B A A A B B \cdots
$$

can be used to check step by step what we are claiming here. For example its FC $\frac{t_{4,2}}{s_{4,2}}=\frac{13}{18}=[1,2,1,1,2]$, which has rank 6, can be expressed as

$$
\phi\left(\frac{13}{18}\right)=L R R L R L \quad \text { or else } \quad \psi\left(\frac{13}{18}\right)=A B A B B B
$$




\subsection{The Farey Shift and Its Relatives}

So far, a sequence in $\{L, R\}^{\mathbb{N}}$ starting with the symbol $R$ has no image in $[0,1]$ with $\phi^{-1}$. Let us make the identification

$$
R^{n_{1}} L^{n_{2}} R^{n_{3}} \cdots=S L^{n_{1}} R^{n_{2}} L^{n_{3}} \cdots \equiv L^{n_{1}} R^{n_{2}} L^{n_{3}} \cdots
$$

and denote by $\Sigma$ the half-space of $\{L, R\}^{\mathbb{N}}$ so obtained. We can write

$$
\Sigma=\{L, R\}^{\mathbb{N}} / S
$$

We see that the map $\phi$ is a bijection between $[0,1]$ and $\Sigma$.

Let $\Phi: \Sigma \rightarrow \Sigma$ be the Farey shift map defined by

$$
\Phi\left(L^{a_{1}} R^{a_{2}} L^{a_{3}} R^{a_{4}} \cdots\right)=L^{a_{1}-1} R^{a_{2}} L^{a_{3}} R^{a_{4}} \cdots
$$

Note that, besides $L^{\infty}$ the only fixed point of $\Phi$ is given by the sequence $L R L R L R \cdots$ which is the image with $\phi$ of $x=[1,1, \cdots]$, the golden mean. This map acts on points in $\mathcal{F}$ by reducing their rank of one unit. For example, since $\phi\left(\frac{13}{18}\right)=L R R L R L$, with the identifications made above we have

$$
\begin{aligned}
& \Phi\left(\phi\left(\frac{13}{18}\right)\right)=R R L R L \equiv L L R L R=\phi\left(\frac{5}{13}\right) \\
& \Phi\left(\phi\left(\frac{5}{13}\right)\right)=L R L R=\phi\left(\frac{5}{8}\right) \\
& \Phi\left(\phi\left(\frac{5}{8}\right)\right)=R L R \equiv L R L=\phi\left(\frac{3}{5}\right) \\
& \Phi\left(\phi\left(\frac{3}{5}\right)\right)=R L \equiv L R=\phi\left(\frac{2}{3}\right) \\
& \Phi\left(\phi\left(\frac{2}{3}\right)\right)=R \equiv L=\phi\left(\frac{1}{2}\right)
\end{aligned}
$$

Let us define the Farey map $F:[0,1] \rightarrow[0,1]$ given by

$$
F(x)= \begin{cases}\frac{x}{1-x}, & \text { if } 0 \leq x \leq \frac{1}{2}, \\ \frac{1-x}{x}, & \text { if } \frac{1}{2}<x \leq 1 .\end{cases}
$$

Its name can be related to the easily verified observation that the set of pre-images $\bigcup_{k=0}^{\ell} F^{-k}\{0\}$ coincides with $\mathcal{F}_{\ell}$ for all $\ell \geq 1$. Note also that the $\ell$-th row of the Farey tree is precisely $F^{-(\ell-1)}\left(\frac{1}{2}\right)$. In particular, this implies that $\bigcup_{k=0}^{\infty} F^{-k}\{0\}=\mathbb{Q} \cap[0,1]$.

Proposition 3.12 Let $\phi:[0,1] \rightarrow \Sigma$ be the coding described above. Then

$$
\Phi \circ \phi=\phi \circ F \text {. }
$$

Proof. If $1 / 2<x \leq 1$ then $a_{1}=1$ and $F(x)=\frac{1}{x}-a_{1}$. If instead $0<x \leq 1 / 2$ then $a_{1}>1$ and $F(x)=1 /\left(\frac{1}{x}-1\right)$. Therefore,

$$
\text { if } x=\left[a_{1}, a_{2}, a_{3}, \cdots\right] \text { then } F(x)=\left[a_{1}-1, a_{2}, a_{3}, \cdots\right] \text {, }
$$

with $\left[0, a_{2}, a_{3}, \cdots\right] \equiv\left[a_{2}, a_{3}, \cdots\right]$. The claim now follows from (3.23) and (3.21). 


\subsubsection{The Gauss and Fibonacci Maps}

The map $F$ has (at least) two induced versions: the first one is the Gauss map $G:[0,1] \rightarrow[0,1]$ already introduced in (2.28), which for $x>0$ can be written as

$$
G(x)=F^{[1 / x]}=\{1 / x\} .
$$

Recall that

$$
\text { if } x=\left[a_{1}, a_{2}, a_{3}, \cdots\right] \text { then } G(x)=\left[a_{2}, a_{3}, \cdots\right] \text {. }
$$

Noting that

$$
G(x)=F^{n}(x) \text { if } x \in A_{n}=\left(\frac{1}{n+1}, \frac{1}{n}\right]
$$

we see that $G$ is obtained by iterating $F$ once plus the number of times necessary to reach the interval $[1 / 2,1]$. The second one is the Fibonacci map $H$ and is defined by iterating $F$ once plus the number of times necessary to reach the interval $[0,1 / 2]$. Let $F_{0}=0, F_{1}=1$ and $F_{n+1}=F_{n}+F_{n-1}$ for $n \geq 1$ be the Fibonacci numbers. Then, for $n \geq 0$,

$$
H(x)= \begin{cases}\frac{F_{2 n+1} x-F_{2 n}}{F_{2 n+1}-F_{2 n+2} x}, & \text { if } x \in B_{2 n}, \\ \frac{F_{2 n+1}-F_{2 n+2} X}{F_{2 n+5} x-F_{2 n+4}}, & \text { if } x \in B_{2 n+1},\end{cases}
$$

with

$$
B_{2 n}=\left[\frac{F_{2 n}}{F_{2 n+1}}, \frac{F_{2 n+2}}{F_{2 n+3}}\right), \quad B_{2 n+1}=\left(\frac{F_{2 n+3}}{F_{2 n+4}}, \frac{F_{2 n+1}}{F_{2 n+2}}\right] .
$$

In this case it is easy to check that if $x=\left[a_{1}, a_{2}, a_{3}, \cdots\right]$ then

$$
H(x)=\left[a_{r}-1, a_{r+1}, \cdots\right] \text {, where } r=\min \left\{i: a_{i}>1\right\} .
$$

A sketch of the map $F$ along its induced versions $G$ and $H$ is given in Figure 3.

Given $M=\left(\begin{array}{ll}a & b \\ c & d\end{array}\right)$ we may define the Möbius transformation

$$
x \rightarrow \hat{M}(x):=\frac{a x+b}{c x+d}
$$

By the above, given $x \in[0,1 / 2]$ the point $\phi^{-1} \circ L^{-1} \circ \phi(x)$ is but $F(x)$ and for $x \in[0,1 / 2]$ we have $F(x)=\phi^{-1} \circ L^{-1} \circ \phi(x)=\hat{L}^{-1}(x)$ (recall that $L^{-1}=\left(\begin{array}{cc}1 & 0 \\ -1 & 1\end{array}\right)$ ). But what happens if $x \in(1 / 2,1]$ so that $a_{1}=1$ ?

To see this we put

$$
I_{0} \equiv L=\left(\begin{array}{ll}
1 & 0 \\
1 & 1
\end{array}\right) \text { and } I_{1}=S R=L S=\left(\begin{array}{ll}
0 & 1 \\
1 & 1
\end{array}\right)
$$

We have

$$
I_{1} R^{n_{1}} L^{n_{2}} R^{n_{3}} \cdots=I_{1} L^{n_{1}} R^{n_{2}} L^{n_{3}} \cdots
$$

Therefore, noting that $I_{1}^{-1}=\left(\begin{array}{cc}-1 & 1 \\ 1 & 0\end{array}\right)$, for $x \in(1 / 2,1]$ we have $F(x)=\phi^{-1} \circ I_{1}^{-1} \circ \phi(x)=\hat{I}_{1}^{-1}(x)$. To summarize we can represent the action of $F$ as

$$
F(x)= \begin{cases}\hat{I}_{0}^{-1}(x), & \text { if } x \in[0,1 / 2], \\ \hat{I}_{1}^{-1}(x), & \text { if } x \in(1 / 2,1],\end{cases}
$$



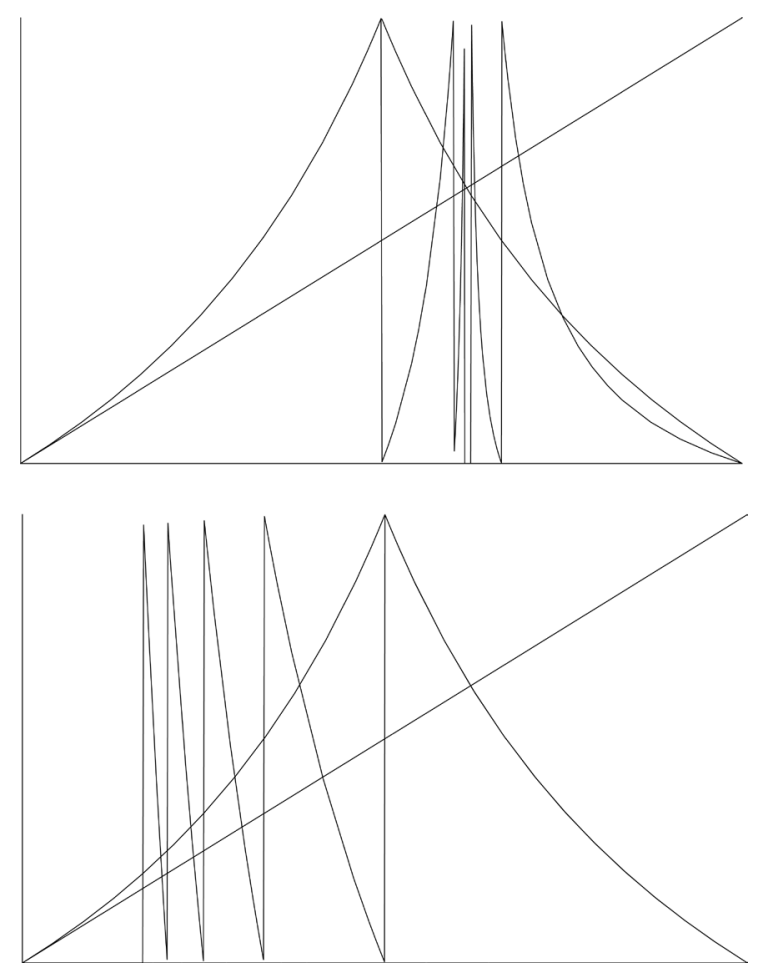

Figure 3. The Farey map and its induced Fibonacci (upper) and Gauss (lower) maps.

that of $G$ as

$$
G(x)=\hat{I}_{1}^{-1} \circ \hat{I}_{0}^{-(n-1)}(x), \quad x \in A_{n} .
$$

and that of $H$ as

$$
H(x)=\hat{I}_{0}^{-1} \circ \hat{I}_{1}^{-(n-1)}(x), \quad x \in B_{n} .
$$

\subsubsection{The Modified Farey Map}

Finally we introduce the modified Farey map $\tilde{F}:[0,1] \rightarrow[0,1]$ given by

$$
\tilde{F}(x)= \begin{cases}\frac{x}{1-x}, & \text { if } 0 \leq x \leq \frac{1}{2}, \\ 2-\frac{1}{x}, & \text { if } \frac{1}{2}<x \leq 1 .\end{cases}
$$

This map preserves orientation and has two indifferent fixed points, at 0 and 1 . The advantage of using $\tilde{F}$ instead of $F$ is that one can retrace the path from a leaf $x \in \mathcal{F}$ back to the root $1 / 2$. More precisely, for $x \in \mathcal{F}$ let (cf. Proposition 3.8) $X=L \prod_{i} M_{i}$ be the element which uniquely represents $x$ in $\mathcal{U}$. Then one easily sees that the following rule is in force: if $\tilde{F}^{(i-1)}(x)<1 / 2$ then $M_{i}=L, \tilde{F}^{(i-1)}(x)>1 / 2$ then $M_{i}=R$, for $i=1, \cdots, k$ with $k=\operatorname{rank}(x)$ so that $\tilde{F}^{k}(x)=1 / 2$.

\section{The Minkowski Question Mark}

Given a number $x \in(0,1)$ with continued fraction expansion $x=\left[a_{1}, a_{2}, a_{3}, \cdots\right]$, one may ask what is the number obtained by interpreting the sequence $\phi(x)$ (see (3.14)) as the binary expansion of a real number in $(0,1)$. The number so obtained is denoted $?(x)$ and writes

$$
?(x)=0 . \underbrace{00 \cdots 0}_{a_{1}-1} \underbrace{11 \cdots 1}_{a_{2}} \underbrace{00 \cdots 0}_{a_{3}} \cdots
$$


or, which is the same,

$$
?(x)=\sum_{k \geq 1}(-1)^{k-1} 2^{-\left(a_{1}+\cdots+a_{k}-1\right)} .
$$

For instance $?(1 / n)=1 / 2^{n-1}$, for all $n \geq 1$ (see Figure 4). Setting $?(0)=0$ and $?(1)=1$ one has the following properties for the function $\quad$ : $[0,1] \rightarrow[0,1]$ (see [13]-[16]):

- ? $(x)$ is strictly increasing from 0 to 1 and Hölder continuous of exponent $\beta=\frac{\log 2}{2 \log (\sqrt{5}+1) / 2}$;

- $x$ is rational iff $?(x)$ is of the form $k / 2^{s}$, with $k$ and $s$ integers;

- $x$ is a quadratic irrational iff $?(x)$ is a (non-dyadic) rational;

- $?(x)$ is a singular function: its derivative vanishes Lebesgue-almost everywhere.

The following additional properties easily follow from the definition.

Lemma 4.1 ? $(x)$ satisfies the functional equations

$$
\begin{gathered}
?(1-x)=?(1)-?(x) \\
?(x)=\frac{1}{2} ?\left(\frac{x}{1-x}\right), \quad 0 \leq x \leq 1 / 2
\end{gathered}
$$

Proof. Assuming that $x \in[1 / 2,1]$ we write $x=1 /(y+1)$ with $y \in[0,1]$ and $1-x=y /(y+1) \in[0,1 / 2]$. Setting moreover $y=\left[a_{1}, a_{2}, \cdots\right]$ we have $x=\left[1, a_{1}, a_{2}, \cdots\right]$ and $1-x=\left[1+a_{1}, a_{2}, \cdots\right]$. The assertion now follows by direct application of (4.2).

Let us now see how ? acts on Farey fractions. We have already seen that

$$
?\left(\frac{1}{2}\right)=?\left(\frac{0+1}{1+1}\right)=\frac{1}{2}[?(0)+?(1)]=\frac{1}{2}
$$

More generally, for any pair $a / b$ and $a^{\prime} / b^{\prime}$ of consecutive Farey fractions the function ? equates their child to the arithmetic average:

$$
?\left(\frac{a+a^{\prime}}{b+b^{\prime}}\right)=\frac{1}{2}\left[?\left(\frac{a}{b}\right)+?\left(\frac{a^{\prime}}{b^{\prime}}\right)\right]
$$

One sees that the function ? maps the Farey tree $\mathcal{F}$ to the dyadic tree $\mathcal{D}$ defined as follows: having fixed $\ell \geq 1$, let $\mathcal{D}_{\ell}$ be the ascending sequence of fractions of the form $k / 2^{\ell-1}, k=0,1, \cdots, 2^{\ell-1}$. We have

$$
\mathcal{D}_{1}=\left(\frac{0}{1}, \frac{1}{1}\right), \quad \mathcal{D}_{2}=\left(\frac{0}{1}, \frac{1}{2}, \frac{1}{1}\right), \quad \mathcal{D}_{3}=\left(\frac{0}{1}, \frac{1}{4}, \frac{1}{2}, \frac{3}{4}, \frac{1}{1}\right)
$$

and so on. Then $\mathcal{D}$ is the same graph as $\mathcal{F}$ with the $\ell$-th row replaced by $\mathcal{D}_{\ell+1} \backslash \mathcal{D}_{\ell}$. An immediate consequence of the fact that $?(\mathcal{F})=\mathcal{D}$ is that $?(x)$ is the asymptotic distribution function of the sequence of Farey fractions:

Theorem 4.2 Since

$$
x=\lim _{\ell \rightarrow \infty} \frac{\#\left\{\frac{a}{b} \in \mathcal{D}_{\ell+1}: \frac{a}{b} \leq x\right\}}{2^{\ell}}
$$

then

$$
?(x)=\lim _{\ell \rightarrow \infty} \frac{\#\left\{\frac{a}{b} \in \mathcal{F}_{\ell+1}: \frac{a}{b} \leq x\right\}}{2^{\ell}} .
$$

Remark 4.3 This result can be also deduced as a consequence of a more general result obtained in [17] using a suitable enumeration of the rationals in $(0,1)$. As for the convergence of the atomic measure concentrated on $\mathcal{F}_{n}$ to d? see [11] and [18].

As a further immediate consequence we get that the Fourier-Stieltjes coefficients of ? $(x)$ are as in the following 


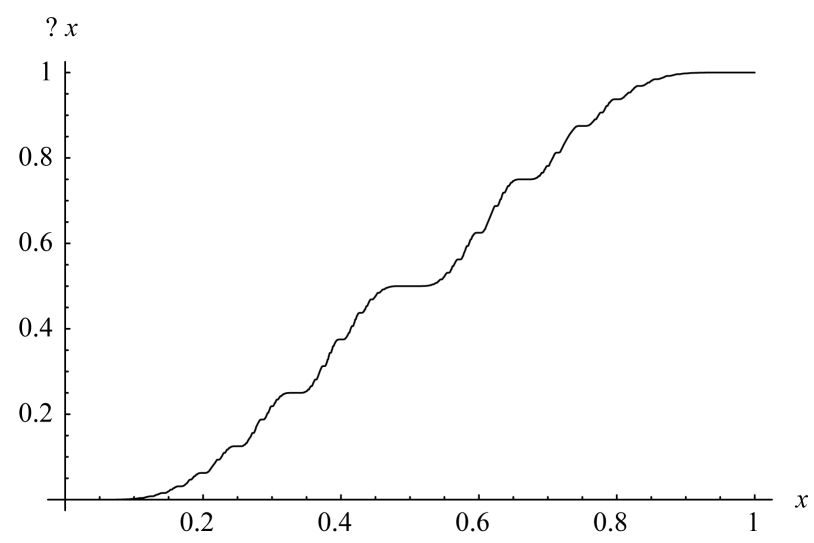

Figure 4. The Minkowski ? function.

\section{Corollary 4.4 Let}

$$
c_{n}=\int_{0}^{1} \mathrm{e}^{2 \pi i n x} \mathrm{~d} ?(x)
$$

then

$$
C_{n}=\lim _{\ell \rightarrow \infty} \frac{1}{2^{\ell}} \sum_{\frac{a}{b} \in \mathcal{F}_{\ell+1}} e^{2 \pi i n \frac{a}{b}} .
$$

Finally, a short reflection using the definition (4.1) shows that ? conjugates the Farey map $F$ and the modified Farey map $\tilde{F}$ to the tent map

$$
T(x)= \begin{cases}2 x, & \text { if } 0 \leq x<1 / 2, \\ 2(1-x), & \text { if } 1 / 2 \leq x \leq 1 .\end{cases}
$$

and the doubling map $D(x)=2 x \bmod 1$, respectively. Indeed, for any $\omega=\omega_{1} \omega_{2} \cdots$ with $\omega_{i} \in\{0,1\}$ we have

and

$$
T^{i}(0.00 \cdots 01 \omega)=0.00 \cdots 01 \omega \text { for } i \leq k
$$

$$
T(0.1 \omega)=0 . \bar{\omega}
$$

where $\bar{\omega}=\bar{\omega}_{1} \bar{\omega}_{2} \cdots$ and $\bar{\omega}_{i}=1-\omega_{i}$. A similar reasoning applies for $D$. Putting together the above, (3.25) and (4.1) we then get the following commutative diagrams

\section{Theorem 4.5}

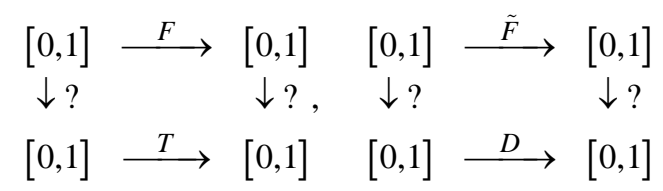

This implies that the measure $d ?(x)$ is invariant under both maps $F$ and $\tilde{F}$, and its entropy is equal to $\log 2$. This makes $d ?(x)$ the measure of maximal entropy for $F$ and $\tilde{F}$. Being zero at every rational point $d$ ? is of course singular w.r.t. Lebesgue. More specifically, $d$ ? is concentrated on a subset $X \subset[0,1]$ having Hausdorff dimension $\alpha \approx 0.875$ (see [14]). In view of (3.25), the above has the following straightforward consequence

Lemma 4.6 If $x$ is drawn from $[0,1]$ according to the singular measure $d ?(x)$, then the partial quotients $\left(a_{i}\right)_{i \geq 1}$ of $x=\left[a_{1}, a_{2}, \cdots\right]$ form a sequence of i.i.r.v.'s with $\operatorname{Prob}\left(a_{i}=n\right)=2^{-n}$.

It is moreover easy to realize that $F$ and $\tilde{F}$ have also absolutely continuous (not normalizable) invariant measures, with densities $1 / x$ and $1 / x(1-x)$, respectively.

Finally, the conjugacy of Theorem 4.5 has been used in [19] to construct a correspondence between the parameter spaces of $\alpha$-continued fraction transformations and unimodal maps. 


\section{Transfer Operators and Partition Functions}

To a given matrix $M=\left(\begin{array}{ll}a & b \\ c & d\end{array}\right) \in \mathcal{U}$ and complex parameter $q$ one can associate the positive operator $\pi_{q}(M)$ acting on the right as [20]

$$
\pi_{q}(M) f(x):=(c x+d)^{-2 q} f\left(\frac{a x+b}{c x+d}\right)=\left|\hat{M}^{\prime}(x)\right|^{q} \cdot f(\hat{M}(x)) .
$$

For example we have

$$
\pi_{q}(S) f(x)=\frac{1}{x^{2 q}} f\left(\frac{1}{x}\right) .
$$

The operator $\mathcal{P}_{q}$ associated in this way to the map $([0,1], F)$ turns out to be the transfer operator acting as

$$
\mathcal{P}_{q} f(x):=\left[\pi_{q}\left(I_{0}\right)+\pi_{q}\left(I_{1}\right)\right] f(x)=\frac{1}{(x+1)^{2 q}}\left[f\left(\frac{x}{x+1}\right)+f\left(\frac{1}{x+1}\right)\right] .
$$

Of special significance is the (Perron-Frobenius) operator $\mathcal{P}_{1}$ which satisfies

$$
\int_{0}^{1} g \circ F(x) f(x) \mathrm{d} x=\int_{0}^{1} g(x) \mathcal{P}_{1} f(x) \mathrm{d} x
$$

and has norm at most one in the Banach space $L^{1}([0,1], \mathrm{d} x)$. A function $h$ is the density of an absolutely continuous invariant measure for $F$ if and only if $\mathcal{P}_{1} h=h$. In this case we find $h(x)=1 / x$, which however does not lie in $L^{1}([0,1]$, $\mathrm{d} x)$ (see [21]).

Let $f$ be an eigenfunction of $\mathcal{P}_{q}$ analytic in the half-plane $\operatorname{Re} x>0$. It satisfies

and also

$$
\lambda f(x)=\mathcal{P}_{q} f(x)=\left(\frac{1}{x+1}\right)^{2 q}\left[f\left(\frac{x}{x+1}\right)+f\left(\frac{1}{x+1}\right)\right]
$$

$$
\pi_{q}(S) f(x)=f(x)
$$

Therefore the eigenvalue equation is equivalent to the three-term equation

$$
\lambda f(x)=f(x+1)+\frac{1}{x^{2 q}} f\left(1+\frac{1}{x}\right)
$$

which is a generalisation of the Lewis functional equation (with $\lambda=1$ ) studied in number theory (see [20] [22]). The study of this generalized equation has been initiated in [23].

Remark 5.1 In the context of the thermodynamic formalism, once a one-sided shift $\Phi: \Sigma \rightarrow \Sigma$ and a potential function $\varphi \in C(\Sigma)$ are given one defines a transfer operator $\mathcal{L}_{\varphi}$ on $C(\Sigma)$ by

$$
\mathcal{L}_{\varphi} g(\xi)=\sum_{\eta \in \Phi^{-1} \xi} \mathrm{e}^{\varphi(\eta)} g(\eta)
$$

which plays a key role in the study of equilibrium states for $(\Sigma, \Phi, \varphi)$ and their properties [24] [25]. In particular, one defines

$$
\operatorname{var}_{k} \varphi=\sup \left\{\left|\varphi(\xi)-\varphi\left(\xi^{\prime}\right)\right|: \xi_{i}=\xi_{i}^{\prime}, 0<i \leq k\right\}
$$

and it turns out that if $\operatorname{var}_{k} \varphi$ decays exponentially then there is a unique mixing equilibrium state.

Relying on the above discussion it is now easy to see that $\phi_{*} \mathcal{P}_{q}=\mathcal{L}_{\varphi}$ with

$$
\varphi(\xi)=-2 q \log \left(1+\phi^{-1}(\xi)\right) .
$$

In order to compute $\operatorname{var}_{k} \varphi$ we have to consider points sharing the same path up to the $k$-th row of $\mathcal{F}$. Take for instance $\xi=L^{\infty}$ and $\xi^{\prime}=L^{k} R \cdots$. Then a short reflection yields, for $q \neq 0$,

$$
\left|\varphi(\xi)-\varphi\left(\xi^{\prime}\right)\right| \geq \frac{C}{k}
$$


We therefore see that although $\operatorname{var}_{k} \varphi \rightarrow 0$ (so that $\varphi$ is uniformly continuous) $\varphi$ it is not even of summable variation. This entails that $(\Sigma, \Phi, \varphi)$ has indeed two equilibrium states, thus exhibiting a phase transition (see [26]).

Next, we express the $n$-th iterate of $\mathcal{P}_{q}$ as

$$
\mathcal{P}_{q}^{n} f(x)=\sum_{\omega \in\{0,1\}^{n}}\left[(1+x) \prod_{i=1}^{n-1}\left(1+\hat{I}_{\omega_{i} \cdots \omega_{1}}(x)\right)\right]^{-2 q} f\left(\hat{I}_{\omega_{n} \cdots \omega_{1}}(x)\right)
$$

where $I_{\omega_{i} \cdots \omega_{1}}:=I_{\omega_{i}} \cdots I_{\omega_{1}}$. We have $\hat{I}_{\omega_{i} \cdots \omega_{1}}(x)=\hat{I}_{\omega_{i}} \circ \hat{I}_{\omega_{i-1} \cdots \omega_{1}}(x)$ so that, in particular, putting $x=0$ we get

$$
\hat{I}_{0}(0)=0, \quad \hat{I}_{1}(0)=1
$$

and

$$
\text { if } \hat{I}_{\omega_{i-1} \cdots \omega_{1}}(0)=\frac{a}{b} \quad \text { then } \hat{I}_{\omega_{i} \cdots \omega_{1}}(0)=\left\{\begin{array}{l}
\frac{a}{a+b}, \text { if } \omega_{i}=0, \\
\frac{b}{a+b}, \text { if } \omega_{i}=1 .
\end{array}\right.
$$

Lemma 5.2 Let $\left(h_{n}\right)_{n \geq 1}$ be the sequence of functions defined by $h_{1} \equiv 1$ and

$$
h_{n}(\omega):=\prod_{i=1}^{n-1}\left(1+\hat{I}_{\omega_{i} \cdots \omega_{1}}(0)\right), \omega \in\{0,1\}^{n-1}, n>1 .
$$

For each fixed $n \geq 1$ we have that $h_{n}$ determines a bijection between $\{0,1\}^{n-1}$ and the set of denominators of the elements of $\mathcal{F}_{n} \backslash\left\{\frac{0}{1}\right\}$ (considered as an ordered set).

Proof. The proof is just a straightforward verification. Suppose for instance that $\omega=0^{k-1} 1 u$ with $u \in\{0,1\}^{\ell-1}$, so that $k+\ell=n$. Then by (5.9) and (5.10) $h_{n}(\omega)$ is given by a product with $\ell$ factors of the type $(1+1)\left(1+\frac{1}{2}\right) \cdots\left(1+\frac{a}{b}\right)\left(1+\frac{r}{a+b}\right)=a+b+r$ where $r=a$ if $u_{\ell-1}=0, r=b$ otherwise. The result now readily follows by lemma 3.6.

Remark 5.3 The rank of the elements of $\mathcal{F}_{n} \backslash\left\{\frac{0}{1}\right\}$ with denominator $h_{n}(\omega)$ is given by $\ell=n-\min \left\{1 \leq i<n, \omega_{i}=1\right\}$ with the convention $\min \varnothing=n$. The smallest of the above denominators is 1 , it has rank 0 and is obtained as $h_{n}\left(0^{n-1}\right)$. The two largest ones are equal to the $(n+1)$-st Fibonacci number $f_{n+1}=\frac{1}{\sqrt{5}}\left[\left(\frac{1+\sqrt{5}}{2}\right)^{n+1}-\left(\frac{1-\sqrt{5}}{2}\right)^{n+1}\right]$. They are symmetrical w.r.t $\frac{1}{2}$, have rank $n-1$ and are obtained as $h_{n}\left(101^{n-3}\right)$ and $h_{n}\left(1^{n-1}\right)$, respectively. More generally, it is not difficult to see that the following equivalence is in force: suppose that the element $\frac{a}{b} \in \mathcal{F}_{n}$ has rank $\ell>1$ so that $b=h_{n}\left(0^{n-\ell-1} 1 x u\right)$ for some $x \in\{0,1\}$ and $u \in\{0,1\}^{\ell-2}$, then the same denominator $b$, but corresponding to the symmetrical fraction $1-\frac{a}{b}$, is obtained as $b=h_{n}\left(0^{n-\ell-1} 1 \bar{x} u\right)$ with $\bar{x}=1-x$.

A direct consequence of the above lemma is the following

\section{Theorem 5.4}

$$
\mathcal{P}_{q}^{n} 1(0)=2 \sum_{\frac{a}{b} \in \mathcal{F}_{n} \backslash\left\{\frac{0}{1}\right\}} b^{-2 q}
$$

Remarkably, the above sum is equal to the partition function $Z_{n-1}(2 q)$ at (inverse) temperature $2 q$ of the number-theoretical spin chain introduced by Andreas Knauf in [27]. For $\operatorname{Re} q>1$ we have (see [28])

$$
\lim _{n \rightarrow \infty} \mathcal{P}_{q}^{n} 1(0)=2 \frac{\zeta(2 q-1)}{\zeta(2 q)}=2 \sum_{k=1}^{\infty} \frac{\varphi(k)}{k^{2 q}} .
$$


Note that for $q=1$ the above limit diverges. This reflects the fact that the invariant density for the Farey map $F$, that is the fixed point of the operator $\mathcal{P}_{1}$, is the function $1 / x$.

Let us define the pressure function $p(q)$ as

$$
p(q):=\lim _{n \rightarrow \infty} \frac{1}{n} \log \mathcal{P}_{q}^{n} 1(0)
$$

Since the sum in Thm. 5.4 has $2^{n-1}$ terms we see that $p(0)=\log 2$ (this is the topological entropy of the map $F$ ). More generally let $\left\{d_{i, n}, i=1, \ldots, 2^{n-1}\right\}$ denote the sequence of denominators of the elements of $\mathcal{F}_{n} \backslash\left\{\frac{0}{1}\right\}$ when the latters are arranged in increasing order in $[0,1]$, so that

$$
Z_{n-1}(2 q)=\frac{1}{2} \mathcal{P}_{q}^{n} 1(0)=\sum_{i=1}^{2^{n-1}} d_{i, n}^{-2 q}
$$

The ratio $Z_{n-1}(2 q) / 2^{n-1}$ can be interpreted as the moment of order $-2 q$ of the size of the denominators in $\mathcal{F}_{n} \backslash\left\{\frac{0}{1}\right\} . Z_{n}(2 q)$ is plainly non-increasing and for $q \leq 0$ satisfies $Z_{n}(2 q) \geq 2^{n}$. Moreover we have

$$
Z_{n}(2 q)=Z_{n-1}(2 q)+\sum_{i=1}^{2^{n-1}}\left(d_{i-1, n}+d_{i, n}\right)^{-2 q}=\sum_{i=1}^{2^{n-1}} d_{i, n}^{-2 q}\left[1+\left(1+\frac{d_{i-1}}{d_{i, n}}\right)^{-2 q}\right]
$$

with $d_{0, n} \equiv 1$ for all $n$. Noting that $\max _{i}\left\{\frac{d_{i-1, n}}{d_{i, n}}\right\}=n$ we get for $q>0$

$$
Z_{n}(2 q) \geq\left[1+\left(\frac{1}{1+n}\right)^{2 q}\right] Z_{n-1}(2 q)
$$

Since $Z_{0}(2 q)=1$ this yields

$$
Z_{n}(2 q) \geq\left[1+\left(\frac{1}{1+n}\right)^{2 q}\right]^{n}, \quad n \geq 1
$$

Thus, for all $q \in \mathbb{R}$,

$$
p(q)=\lim _{n \rightarrow \infty} \frac{1}{n} \log 2 Z_{n-1}(2 q) \geq 0 .
$$

In addition, since $p(q)$ is non-increasing and $p(1)=0$ (because the spectral radius of $P_{1}$ is 1 , see above) we have $p(q)=0$ for $q \geq 1$. Note that the same conclusion follows at once from the fact that $Z_{n}(2 q)$ is finite for $\operatorname{Re} q>1$ (see (11)).

Remark 5.5 It holds $p(q)=-2 q f(2 q)$ where $f(\beta)$ is the free energy of the Knauf model. In the context of thermodynamic formalism the pressure $p(q)$ is a central object. In particular it is used as a generator of averages: its first derivative $p^{\prime}(q)$, wherever it exists, yields the mean of the function $\varphi(x) / q=-2 \log (1+x)$ w.r.t. the equilibrium measure $\mu_{q}$, which can be defined as the weak *-limit point of atomic measures supported on periodic points of $\mathrm{F}$ weighted with the function $\mathrm{e}^{\varphi}$ [24]. Note that $p^{\prime}(q)=0$ for $q>1$ and $p^{\prime}(q) \nearrow 0$ as $q \nearrow 1$. On the other hand we have already seen that $p(0)=\log 2$ and $\mu_{0}$ is called measure of maximal entropy. Higher derivatives of $p(q)$ are connected to (sums of) higher correlation functions, see [25] [29].

Let us now study the asymptotic behaviour of $Z_{n}(2 q)$ for $q=1$. To this end, we notice that if, instead of $x=0$, we evaluate the iterate $\mathcal{P}_{q}^{n} 1$ at $x=1$, all sequences $\omega \in\{0,1\}^{n}$ in (5.8) yield paths which end up at the same row of the Farey tree. The same argument leading to Theorem 5.4 now yields the following

Corollary 5.6

$$
\mathcal{P}_{q}^{n} 1(1)=2 \sum_{\operatorname{rank}\left(\frac{a}{b}\right)=n} b^{-2 q}, \quad n \geq 1 .
$$


By Thm. 5.4 and (5.16) we obtain

$$
\mathcal{P}_{q}^{n} 1(0)=1+\sum_{\ell=0}^{n-1} \mathcal{P}_{q}^{\ell} 1(1)
$$

so that we can directly apply the results obtained by Thaler in [30] to get ${ }^{4}$

Lemma 5.7

$$
\mathcal{P}_{1}^{n} 1(0)=1+\sum_{\ell=0}^{n-1} \mathcal{P}_{1}^{\ell} 1(1)=2 Z_{n-1}(2) \sim \frac{n}{\log n} .
$$

Lastly, noting that

$$
\mathcal{P}_{q} 1(x)=2(1+x)^{-2 q} \text { and } \mathcal{P}_{q}^{2} 1(x)=2\left[(1+2 x)^{-2 q}+(2+x)^{-2 q}\right]
$$

one may then use $\hat{I}_{\omega_{i} \cdots \omega_{1}}(x)=\hat{I}_{\omega_{i}} \circ \hat{I}_{\omega_{i-1} \cdots \omega_{1}}(x)$, along with Lemma 3.6, to proceed inductively with $f \equiv 1$ in (5.8), and obtain the following general expression for $\mathcal{P}_{q}^{\ell} 1(x)$ with $x \in \mathbb{R}_{+}$.

Theorem 5.8 For all $x \in \mathbb{R}_{+}$and $n \geq 1$ we have

$$
\mathcal{P}_{q}^{n} 1(x)=2 \sum_{\operatorname{rank}\left(\frac{a}{b}\right)=n-1}\left[(a+b x)^{-2 q}+(a x+b)^{-2 q}\right]
$$

We refer to [31] for further generalisations and applications (see also [32]).

\section{The Partition Function for Negative Integer Temperatures}

Finally, we compute the value of the partition function $Z_{n}$ for some some specific value of the temperature. Related results are discussed in [33] (see also [34]).

Lemma 5.9 We have, for all $n \geq 1$,

$$
\begin{gathered}
Z_{n}(0)=2^{n}, \\
Z_{n}(-1)=3^{n}, \\
Z_{n}(-2)=\frac{1}{\sqrt{17}}\left[\left(\frac{5+\sqrt{17}}{2}\right)^{n+1}-\left(\frac{5-\sqrt{17}}{2}\right)^{n+1}\right]
\end{gathered}
$$

Proof. The first identity is trivial. The second one follows immediately from $Z_{0}(-1)=1$ along with (5.14), which gives the recursion $Z_{n}(-1)=3 Z_{n-1}(-1)$. As for the third one, we can reason as follows: let us denote $A_{n} \equiv Z_{n}(-2)=\sum_{i=1}^{2^{n}} d_{i, n}^{2}$ and $B_{n}=\sum_{i=1}^{2^{n}} d_{i-1, n} d_{i, n}$. Then (5.14) yields $A_{n}=3 A_{n-1}+2 B_{n-1}$. Moreover, we have

$$
\begin{aligned}
B_{n} & =\sum_{i=1}^{2^{n}} d_{i-1, n+1} d_{i, n+1}=\sum_{i=1}^{2^{n-1}} d_{i-1, n}\left(d_{i-1, n}+d_{i, n}\right)+\sum_{i=1}^{2^{n-1}}\left(d_{i-1, n}+d_{i, n}\right) d_{i, n} \\
& =\sum_{i=1}^{2^{n-1}}\left(d_{i-1, n}+d_{i, n}\right)^{2}=A_{n}-A_{n-1}
\end{aligned}
$$

This yields the recursion $A_{n+1}=5 A_{n}-2 A_{n-1}$ with $A_{0}=1$ and $A_{1}=5$ and the claim easily follows $\bullet$. The above result indicates a general argument to work out $Z_{n}(-k)$ for any $k \in \mathbb{N}$ : setting $A_{n}^{(k)}=Z_{n}(-k)$ and $B_{n}^{(k, r)}=\sum_{i=1}^{2^{n}} d_{i-1, n}^{r} d_{i, n}^{k-r}$ one has

$$
A_{n}^{(k)}=3 A_{n-1}^{(k)}+\sum_{r=1}^{k-1}\left(\begin{array}{l}
k \\
r
\end{array}\right) B_{n-1}^{(k, r)}
$$

and

\footnotetext{
${ }^{4}$ We say that $a_{n}$ and $b_{n}$ are asymptotically equivalent, denoted as $a_{n} \sim b_{n}$, if the quotient $a_{n} / b_{n}$ tends to unity as $n$ approaches $\infty$.
} 


$$
\begin{aligned}
B_{n}^{(k, r)} & =\sum_{i=1}^{2^{n}} d_{i-1, n+1}^{r} d_{i, n+1}^{k-r} \\
& =\sum_{i=1}^{2^{n-1}}\left[d_{i-1, n}^{r}\left(d_{i-1, n}+d_{i, n}\right)^{k-r}+\left(d_{i-1, n}+d_{i, n}\right)^{r} d_{i, n}^{k-r}\right] \\
& =\sum_{s=0}^{r}\left(\begin{array}{l}
r \\
s
\end{array}\right) B_{n-1}^{(k, s)}+\sum_{s=0}^{k-r}\left(\begin{array}{c}
k-r \\
s
\end{array}\right) B_{n-1}^{(k, r+s)} \\
& =2 A_{n-1}^{(k)}+\sum_{s=1}^{r-1}\left(\begin{array}{l}
r \\
s
\end{array}\right) B_{n-1}^{(k, s)}+2 B_{n-1}^{(k, r)}+\sum_{s=r+1}^{k-1}\left(\begin{array}{c}
k-r \\
s-r
\end{array}\right) B_{n-1}^{(k, s)}
\end{aligned}
$$

This yields a $k$-dimensional recursion

$$
\left(\begin{array}{c}
A_{n}^{(k)} \\
B_{n}^{(k, 1)} \\
\vdots \\
B_{n}^{(k, k-1)}
\end{array}\right)=V_{k}\left(\begin{array}{c}
A_{n-1}^{(k)} \\
B_{n-1}^{(k, 1)} \\
\vdots \\
B_{n-1}^{(k, k-1)}
\end{array}\right)
$$

with $k \times k$ matrix

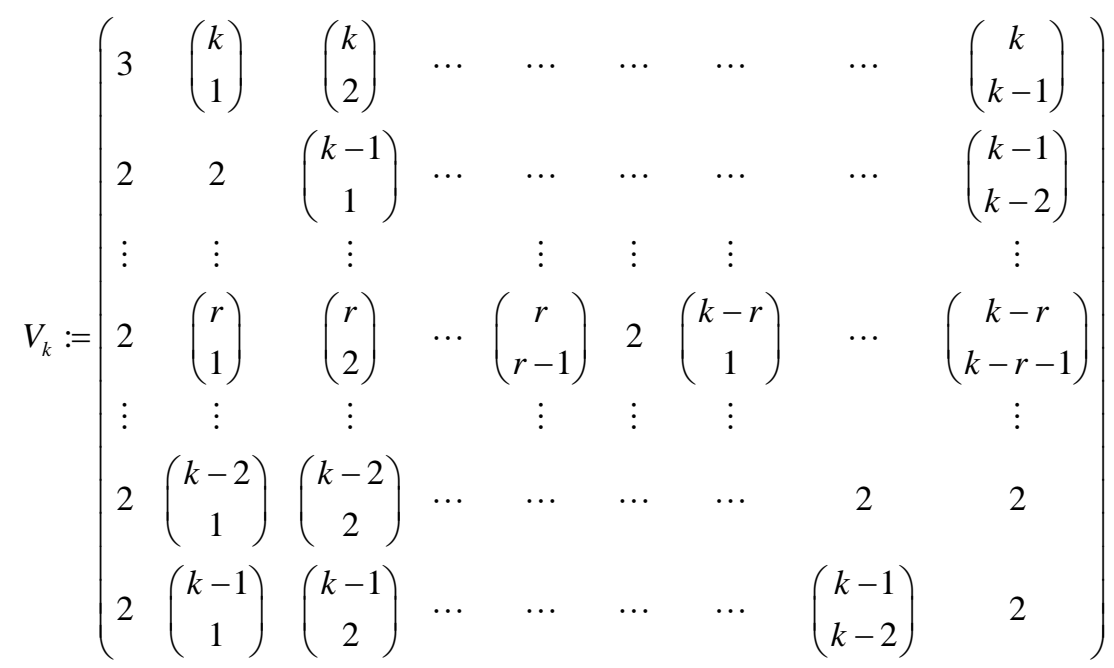

and initial condition

$$
\left(\begin{array}{c}
A_{n}^{(k)} \\
B_{n}^{(k, 1)} \\
\vdots \\
B_{n}^{(k, k-1)}
\end{array}\right)=\mathbf{1}:=\left(\begin{array}{c}
1 \\
1 \\
\vdots \\
1
\end{array}\right)
$$

By Perron-Frobenius theorem the matrix $V_{k}$ has a simple real positive maximal eigenvalue $\lambda_{1}(k)$ whose eigenvector $v_{1}$ has strictly positive components. This immediately yields

$$
p(-k / 2)=\log \lambda_{1}(k) .
$$

More specifically, by the above the exact behaviour of $Z_{n}(-k)$ can be obtained by standard linear algebra. If for instance $V_{k}$ can be diagonalized with spectrum $\operatorname{sp}\left(V_{k}\right)=\left(\lambda_{i}\right)_{1 \leq i \leq k}$ and corresponding eigenvectors $\left(v_{i}\right)_{1 \leq i \leq k}$, then we can expand $\mathbf{1}=\sum_{i} a_{i} v_{i}$ so that (5.18) and (5.19) yield

$$
Z_{n}(-k)=\sum_{i=1}^{k} a_{i} v_{i}^{(1)} \lambda_{i}^{n}, \quad n \geq 0,
$$


where $v_{i}^{(1)}$ denotes the first component of $v_{i}$. On the other hand, as we shall see in the forthcoming example, $V_{k}$ is not always diagonalizable.

Examples. For $2 q=-2$ we find

$$
V_{2}=\left(\begin{array}{ll}
3 & 2 \\
2 & 2
\end{array}\right), \quad \operatorname{sp}\left(V_{2}\right)=\left\{\frac{5+\sqrt{17}}{2}, \frac{5-\sqrt{17}}{2}\right\}
$$

so that by (5.20) $p(-1)=\log \left(\frac{5+\sqrt{17}}{2}\right)$ and using (5.21) one easily recover the result of Lemma 5.9 for $Z_{n}(-2)$. For $2 q=-3$ we get

$$
V_{3}=\left(\begin{array}{lll}
3 & 3 & 3 \\
2 & 2 & 2 \\
2 & 2 & 2
\end{array}\right), \quad \operatorname{sp}\left(V_{3}\right)=\{7,0,0\} .
$$

In this case (5.21) does not hold but one easily finds

$$
Z_{n}(-3)=9 \cdot 7^{n-1}, \quad n>1,
$$

and $p(-3 / 2)=\log 7$.

The case $2 q=-4$ is still different, yielding

$$
V_{4}=\left(\begin{array}{llll}
3 & 4 & 6 & 4 \\
2 & 2 & 3 & 3 \\
2 & 2 & 2 & 2 \\
2 & 3 & 3 & 2
\end{array}\right), \quad \operatorname{sp}\left(V_{4}\right)=\left\{\frac{11+\sqrt{113}}{2}, \frac{11-\sqrt{113}}{2},-1,-1\right\} .
$$

\section{References}

[1] Khinchin, A.Y. (1964) Continued Fractions. The University of Chicago Press, Chicago.

[2] Hardy, G. H. and Wright, E. M. (1979) An Introduction to the Theory of Numbers. Oxford University Press, Oxford.

[3] Hensley, D. (2006) Continued Fractions. World Scientific, Singapore City.

[4] Adamczewski, B. and Allouche, J.P. (2007) Reversal and Palindromes in Continued Fractions. Theoretical Computer Science, 380, 220-237. http://dx.doi.org/10.1016/j.tcs.2007.03.017

[5] Farey, J. (1816) On a Curious Property of Vulgar Fractions. London and Edinburgh Philosophical Magazine and Journal of Science, 47, 385-386.

[6] Appelgate, H. and Onishi, H. (1983) The Slow Continued Fraction Algorithm via $2 \times 2$ Integer Matrices. The American Mathematical Monthly, 90, 443-455. http://dx.doi.org/10.2307/2975721

[7] Lagarias, J.C. (2001) The Farey Shift and the Minkowski ?-Function. Preprint.

[8] Apostol, T. (1976) Modular Functions and Dirichlet Series in Number Theory. Graduate Texts in Mathematic 41, Springer, New York. http://dx.doi.org/10.1007/978-1-4684-9910-0

[9] Bonanno, C. and Isola, S. (2009) A Renormalization Approach to Irrational Rotations. Annali di Matematica Pura ed Applicata, 188, 247-267. http://dx.doi.org/10.1007/s10231-008-0074-5

[10] Alessandri, P. and Berthè, V. (1998) Three Distances Theorem and Combinatorics on Words. L'Enseignement Mathématique, 44, 103-132.

[11] Conze, J.P. and Guivarc’h, Y. (2002) Densité d’orbites d'actions de groupes linéaires et propriétés d'équidistribution de marches aléatoires. In: Burger, M. and Iozzi, A., Eds., Rigidity in Dynamics and Geometry (Cambridge 2000), Springer, Berlin, 39-76.

[12] Knauf, A. (1999) Number Theory, Dynamical Systems and Statistical Mechanics. Reviews in Mathematical Physics, 11, 1027-1060. http://dx.doi.org/10.1142/S0129055X99000325

[13] Salem, R. (1943) On Some Singular Monotone Functions Which Are Strictly Increasing. Transactions of the American Mathematical Society, 53, 427-439. http://dx.doi.org/10.1090/S0002-9947-1943-0007929-6

[14] Kinney, J.R. (1960) A Note on a Singular Function of Minkowski. Proceedings of the American Mathematical Society, 11, 788-794. 
[15] Bonanno, C. and Isola, S. (2009) Orderings of the Rationals and Dynamical Systems. Colloquium Mathematicum, 116, 165-189. http://dx.doi.org/10.4064/cm116-2-3

[16] Alkauskas, G. (2010) The Minkowski Question Mark Function: Explicit Series for the Dyadic Period Function and Moments. Mathematics and Computation, 79, 383-418. http://dx.doi.org/10.1090/S0025-5718-09-02263-7

[17] Viader, P., Paradis, J. and Bibiloni, L. (2001) A New Light on Minkowski’s ?(x) function. Journal of Number Theory, 73, 212-227. http://dx.doi.org/10.1006/jnth.1998.2294

[18] Gouëzel, S. (2009) Local Limit Theorem for Non-Uniformly Partially Hyperbolic Skew-Products and Farey Sequences. Duke Mathematical Journal, 147, 192-284. http://dx.doi.org/10.1215/00127094-2009-011

[19] Bonanno, C., Carminati, C., Isola, S. and Tiozzo, G. (2013) Dynamics of Continued Fractions and Kneading Sequences of Unimodal Maps. Discrete and Continuous Dynamical Systems, 33, 1313-1332. http://dx.doi.org/10.3934/dcds.2013.33.1313

[20] Lewis, J.B. and Zagier, D. (1997) Period Functions and the Selberg Zeta Function for the Modular Group. In: Drouffe, D.M. and Zuber, J.B., Eds., The Mathematical Beauty of Physics, Advanced Series in Mathematical Physics, Vol. 24, World Scientific, River Edge, 83-97.

[21] Isola, S. (2002) On the Spectrum of Farey and Gauss Maps. Nonlinearity, 15, 1521-1539. http://dx.doi.org/10.1088/0951-7715/15/5/310

[22] Lewis, J.B. (1997) Spaces of Holomorphic Functions Equivalent to Even Maass Cusp forms. Inventiones Mathematicae, 127, 271-306. http://dx.doi.org/10.1007/s002220050120

[23] Bonanno, C. and Isola, S. (2014) A Thermodynamic Approach to Two-Variable Ruelle and Selberg Zeta Functions via the Farey Map. Nonlinearity, to appear.

[24] Bowen, R. (1976) Equilibrium States and Ergodic Theory of Anosov Diffeomorphisms. Lecture Notes in Mathematics, 470.

[25] Ruelle, D. (1978) Thermodynamic Formalism. Addison-Wesley Publishing Company, Boston.

[26] Isola, S. (2003) On Systems with Finite Ergodic Degree. Far East Journal of Dynamical Systems, 5, 1-62.

[27] Knauf, A. (1998) The Number-Theoretical Spin Chain and the Riemann Zeros. Communications in Mathematical Physics, 196, 703-731. http://dx.doi.org/10.1007/s002200050441

[28] Knauf, A. (1993) On a Ferromagnetic Spin Chain. Communications in Mathematical Physics, 153, 77-115. http://dx.doi.org/10.1007/BF02099041

[29] Kotani, M. and Sunada, T. (2001) The Pressure and Higher Correlations for an ANOSOV Diffeomorphism. Ergodic Theory and Dynamical Systems, 21, 807-821. http://dx.doi.org/10.1017/S0143385701001407

[30] Thaler, M. (1995) A Limit Theorem for the Perron-Frobenius Operator of Transformations on [0,1] with Indifferent Fixed Points. Israel Journal of Mathematics, 91, 111-127. http://dx.doi.org/10.1007/BF02761642

[31] Degli Esposti, M., Isola, S. and Knauf, A. (2007) Generalized Farey Trees, Transfer Operators and Phase Transitions. Communications in Mathematical Physics, 275, 297-329. http://dx.doi.org/10.1007/s00220-007-0294-3

[32] Boca, F. (2007) Products of Matrices $\left[\begin{array}{ll}1 & 1 \\ 0 & 1\end{array}\right]$ and $\left[\begin{array}{ll}1 & 0 \\ 1 & 1\end{array}\right]$ and the Distribution of Reduced Quadratic Irrationals. Journal für die reine und angewandte Mathematik, 2007, 149-165.

[33] Bonanno, C., Graffi, S. and Isola, S. (2008) Spectral Analysis of Transfer Operators Associated to Farey Fractions. Rendiconti Lincei-Matematica e Applicazioni, 19, 1-23. http://dx.doi.org/10.4171/RLM/505

[34] Contucci, P. and Knauf, A. (1997) The Phase Transition of the Number-Theoretical Spin Chain. Forum Mathematicum, 9, 547-567. http://dx.doi.org/10.1515/form.1997.9.547 